\title{
A Hybrid Structure of Dual Stators and a Pneumatic Spring for Resonance Control in an Air Mount
}

\author{
Hyung-Tae Kim ${ }^{1}$, Cheol-Ho Kim ${ }^{1}$, Sung-Bok Kang ${ }^{1}$, Seok-Jun Moon ${ }^{2}$, Gyu-Seop Lee ${ }^{3}$ \\ ${ }^{1}$ Smart System Research Group, KITECH, CheonAn, South Korea; ${ }^{2}$ Shock and Vibration Team, KIMM, DaeJeon, South Korea; \\ ${ }^{3}$ Structure Dynamic Design Group, RMS Technology, CheonAn, South Korea. \\ Email: htkim@kitech.re.kr, sjmoon@kimm.re.kr,lgs@rmstech.co.kr
}

Received November $19^{\text {th }}, 2012$; revised December $22^{\text {nd }}, 2012$; accepted January $4^{\text {th }}, 2013$

\begin{abstract}
An active device using electromagnetic forces was constructed and examined for the purpose of minimizing the resonance in air mounts of clean rooms. The air mounts are vulnerable to low-frequency resonance due to heavy weight and low stiffness. A hybrid structure of the active device, composed of pneumatic and electromagnetic parts, was developed and tested. The pneumatic parts in the device support heavy weights under the air mounts, and the electromagnetic parts reduce the resonance. The electromagnetic parts are composed of dual stators and an armature, which surround the pneumatic parts. The resonance can decrease when electromagnetic forces are generated in the gaps between the stators and the armature. Four active devices were installed under a 3-ton surface plate for a vibration test apparatus. The vibration was detected by eddy-current sensors. Discrete P Control logic was based on displacement, and embedded in a C6713 DSP. The results from impact tests show that the peak magnitude in the resonance frequency can be reduced to $-10 \mathrm{~dB}$.
\end{abstract}

Keywords: Low Frequency Resonance; Active Vibration Control; Pneumatic Spring; Air Mount

\section{Introduction}

Vibration is a sensitive issue in manufacturing the IT core components used in smart devices. Manufacturing processes require nanometer accuracy, so vibration is a negative factor in maintaining the quality of the components. Conventional manufacturing environmental factors can be controlled such as particles, humidity and temperature, but it is hard to handle vibration, since it passes through solid material. Air mounts are commonly used to isolate and control vibration [1]. A manufacturing machine is installed on the surface plate of an air mount. The surface plate can sustain several tons of machine weight and floats by multiple air springs operated by pneumatic systems. The air mount is efficient in isolating vibration from the floor in a clean room, but weak against low-frequency resonance [2]. Active pneumatic control adjusts the air flow and pressure in the air springs. Various cases have been reported, based on classical and robust control [3,4]. However, pneumatic systems are slow due to mechanical response, so it may be useful to maintain the planar level of a surface plate.

Electromagnetic control may be an option, by applying electromagnetic levitation $[5,6]$. However, electromagnetic force rapidly decreases for wider gaps, and the volume of the electromagnet rapidly becomes larger for that needed to supply stronger force. Electromagnetic actuators usually have insufficient force, so it is hard to apply them exclusively in air mounts. The use of electromagnetic devices in air mounts is aimed at reducing low-frequency resonance, after transient responses when machine vibration occurs on the air mount.

We have been researching these vibration control devices by combining pneumatic and electromagnetic forces. A permanent magnet and coil were installed in an air spring. The settling time was shortened, after experimentally applying active control [7]. The electromagnetic forces were bidirectional, and formed between a coil and a plate-shaped magnet. The electromagnetic parts were inserted into the chamber of an air spring. A second model was also composed of 8 permanent magnets and coils, which were installed outside of the air spring [8]. The second model was targeted at combining magnetic and rheological actuators. A third model in this study was composed of pneumatic parts, dual coils and a ferrite plate and did not contain a permanent magnet. The active devices were installed in an air mount, and the resonance reduction was examined, using a DSP controller in an impact test.

\section{Design of the Active Device}

\subsection{Structures with Permanent Magnets}

For the purpose of coupling pneumatic and electromag- 
netic forces, we have developed two kinds of active devices. An internal type contains the electromagnetic parts in an air chamber of the air spring [9]. A permanent magnet moves in the vertical direction, and is connected to an upper plate, which contacts a surface plate. Electromagnetic force between the gap can be different from a upward and a downward motion. There is a limit to increase of the capacity of the coil, because of the chamber volume. Electric wiring is required through the air spring, so the pneumatic sealing may possibly be weak. Metal components are exposed to moisture in the compressed air, which causes severe corrosion. In contrast in an external type, multiple coil and permanent magnet pairs surround the air spring. Multiple electro-magnets can be arranged around the air spring. The vertical and bidirectional motion is controlled by the electric current, as in the internal type. It is easy to observe the gap, coils and cores, and convenient to assemble and maintain the active device, but the size of the active device can be increased. Figure 1 shows the internal and external types of active devices. Both the internal and external types have electrical polarity, which can change the positive direction of the vertical motion.

\subsection{Structures without Permanent Magnets}

The active device in this research was designed with consideration of the following three conditions. The first is to not use any permanent magnets, while maintaining the ability to apply electromagnetic forces bi-directionally. The second condition is external installation of the electromagnetic parts is, considered for the case of repair. The pneumatic and electromagnetic forces act on the center of the device and can be activated simultaneously. In the first condition, we consider thin ferrite between dual coils. The coils are the stator, and the ferrite is an armature, as in an electric motor. When electric current is driven into the upper stator, electromagnetic force will be formed in the gap between the armature and the upper stator. In contrast, if the electric current flows into the lower stator, the electromagnetic force will act in the lower direction. Upward and downward motion of an armature can be induced by electric current, after amplifying an input signal. The forces between the stators and
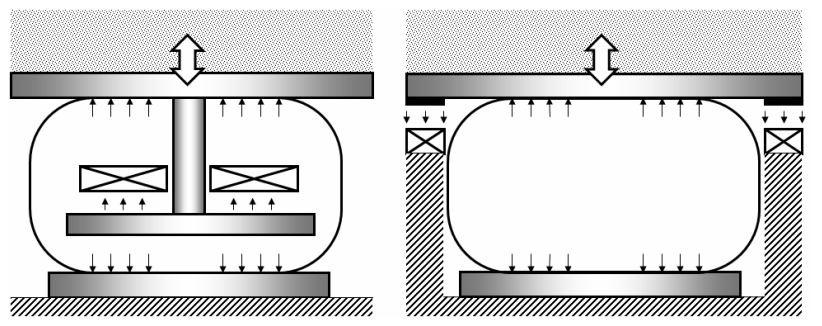

Figure 1. Active devices using electromagnetic forces (a) internal and (b) external. the armature are always attractive, so it is not necessary to check the sign of the current in each stator. For the second condition, we consider wide and ring-shaped stators and armature, which surround an air spring. The air spring is placed in the center of the electromagnetic pairs. For the third condition, the stators are fixed to the floor, and the armature is connected to the upper plate of the air spring. The shape of the electromagnetic parts and the air spring is axial symmetry, and their center is matched, so the upper plate can move in the vertical direction by the pneumatic and electromagnetic forces. The motion range is limited between the gaps of the stators. Figure 2 shows the design concept found in an article by Kazuhide [10] and also proposed by Moon [11].

\subsection{Electromagnetic Analysis [11]}

The proposed concept was concretized and formularized by a permeance model, as shown in Figure 3. We considered the gap, armature material, coil cross-section, number of turns and size of the stator and armature as design parameters. The major performances checked were the forces and the time constant. The model was simulated and analyzed using an FEM tool (ANSYS v11). A quarter model was applied to reduce processing time.

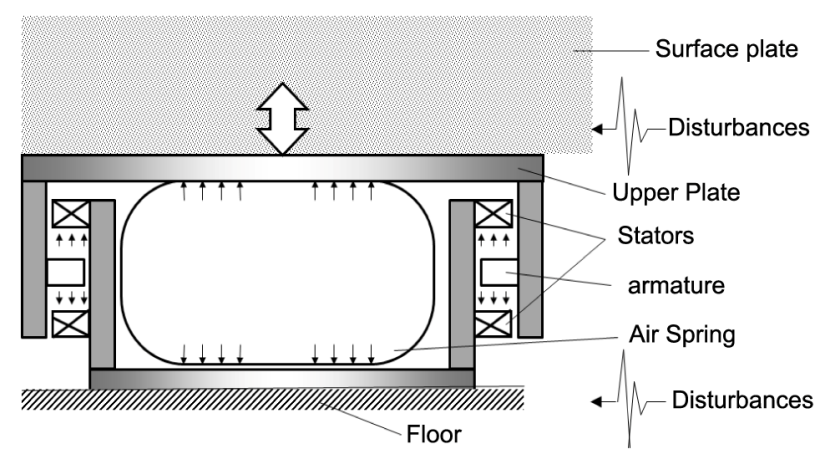

Figure 2. Structure of an active device, with dual stators and an armature.

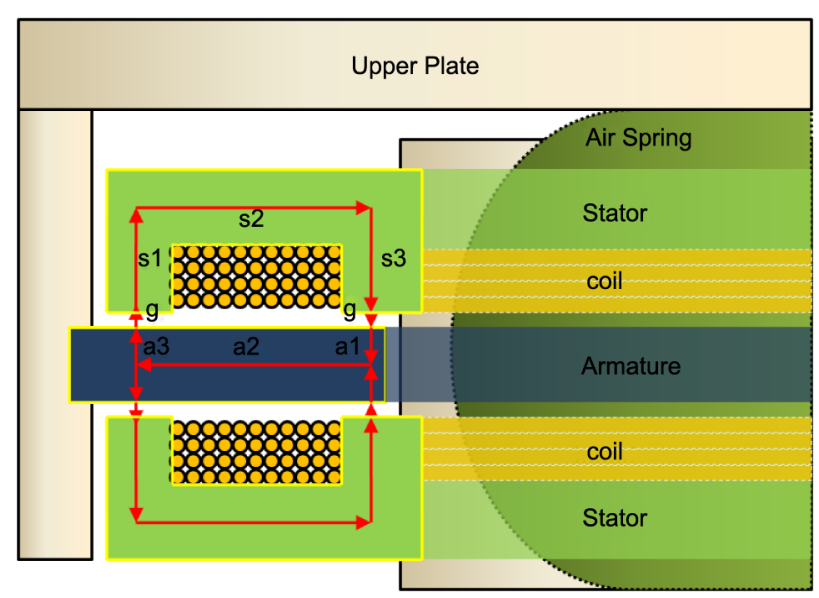

Figure 3. Permeance model and magnetic flux. 
The design parameters were optimized, after simulating the forces and the time constant. Static conditions were assumed for the FEM analysis. The number of nodes and elements were 260,000 and 140,000, respectively. Experiments were carried out while supplying compressed air and sinusoidal driving voltage. The frequency of the voltage ranges from $0.1-10 \mathrm{~Hz}$. When the voltage was applied, the electromagnetic force was measured, using 3 load cells. The control force was constant even after varying the frequency under a sinusoidal voltage. From the simulation and the experimental result, the force was estimated to be $150 \mathrm{~N}$, the inductance $210 \mathrm{mH}$, and the time constant $26 \mathrm{~ms}$.

\section{An Air Mount and Performance Test}

\subsection{Assembly of an Air Mount}

An air mount was constructed with a base frame, a stone surface plate, 4 active devices, 4 gap sensors, 4 throttle valves and 3 height control valves. A base frame was made of cast iron, and placed on the earth. The 4 active devices were installed on the corners of the base frame. A 3-ton stone surface plate was mounted on the active devices. The compressed air was generated in an air supply, and the pressure was adjusted by regulating valves. The amount of regulation was varied manually. The regulated air was transferred to 3 height control valves. The height control valve adjusted the air flow by the vertical motion of a contact point. When the contact point was compressed, the air flow to the active device increased. When the contact point was released, the air flow passed to an exhaust port. Two of the height control valves were installed under the corner of the stone surface plate, and the air from the work port was transferred to an active device. The other was placed under the center point at the center of the opposite edge. The work port was shared with two active devices. Throttle valves were connected between the height control valves and the active devices for setting air pressure in each of the active devices. This mechanism provides mechanical detection of 3 DOF motion in the air mount. Air leak in the pneumatic system can be compensated for and excessive air can decrease in response to vibration and mass movement during motion. Figure 4 shows the arrangement of the pneumatic valves and connection of the air lines.

\subsection{Control of the Active Devices}

Four eddy-current sensors (Santec LS-500) were attached on the corners of the frame and used to detect the gaps between the sensor heads and the surface plate. The initial gaps were set to $3-4 \mathrm{~mm}$ for the base level of the air mount. A DSP controller is a common device for a stand-alone and an embedded system. The DSP controller in this study was composed of a CPU board, a power

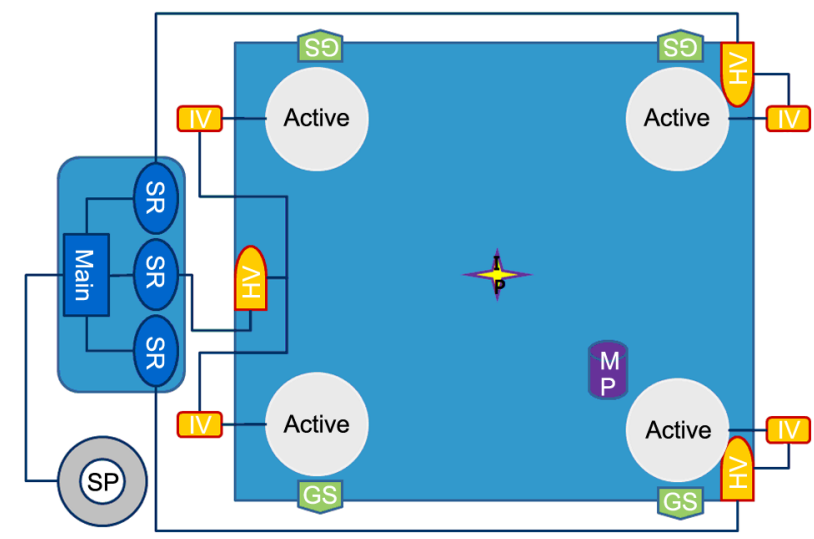

Figure 4. Arrangement of control assemblies in an air mount.

board, an $\mathrm{AD}$ and a $\mathrm{DA}$ converter. The sensor outputs were sampled as 16 bit digital data with $1 \mathrm{MHz}$ sampling rates by the $\mathrm{AD}$ converter (DSignT ADDA12MUX). The sampled signal was transferred to the CPU board (DSignT D.Module C6713). The action of the CPU can be defined using DSP program codes and firmware. The sampled data were filtered, and converted to motion of the surface plate. The sensor voltages in stable status were memorized in the controller for reference inputs. Conventional P control logic was applied, and the control error was calculated from the reference inputs and current sensor outputs. The control output was limited for current saturation. The outputs of the controller were transmitted through a signed $12 \mathrm{bit}, 100 \mathrm{kHz}$ DAC port. The DAC voltages were bipolar and had signed values. Dividers (Daegon Analog Divide B/D) separated pairs of unipolar signals from the bipolar voltages. A pair of unipolar signals had an absolute value in bipolar voltage. Each of the unipolar signals was amplified by a driver (Junus JSP-090-20) to generate a great electromagnetic force in the active device. The control logic and firmware were built using a C-based development tool (TI Code Composer Studio), and loaded into the controller through a JTAG port. The operating modes of the controller were switched using a PC program through the RS-232 port for monitoring the $\mathrm{AD}$ ports, recording sensor data, setting reference inputs, controlling the vibration and testing active devices. Figure 5 shows the signal flow of the controller and Figure 6 shows the electrical connection diagram of the air mount.

\subsection{Experiment}

Impulse responses were observed, to test the performance of vibration control. The center of the surface plate was impacted by a hammer (PCB 086D50). The gap sensor outputs were monitored by a 4-channel oscilloscope (Tek 2014B). An accelerometer (PCB 3713D1FD $3 \mathrm{G})$ was attached between the center and one of the cor- 


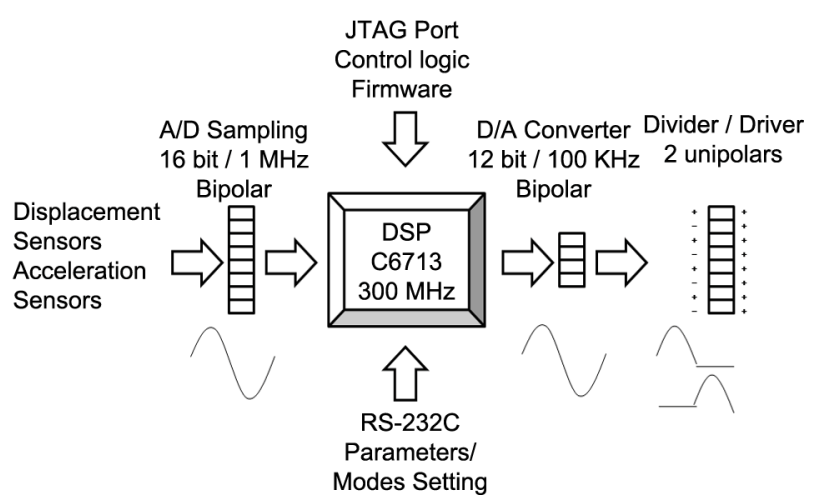

Figure 5. Signal flow of a DSP controller.

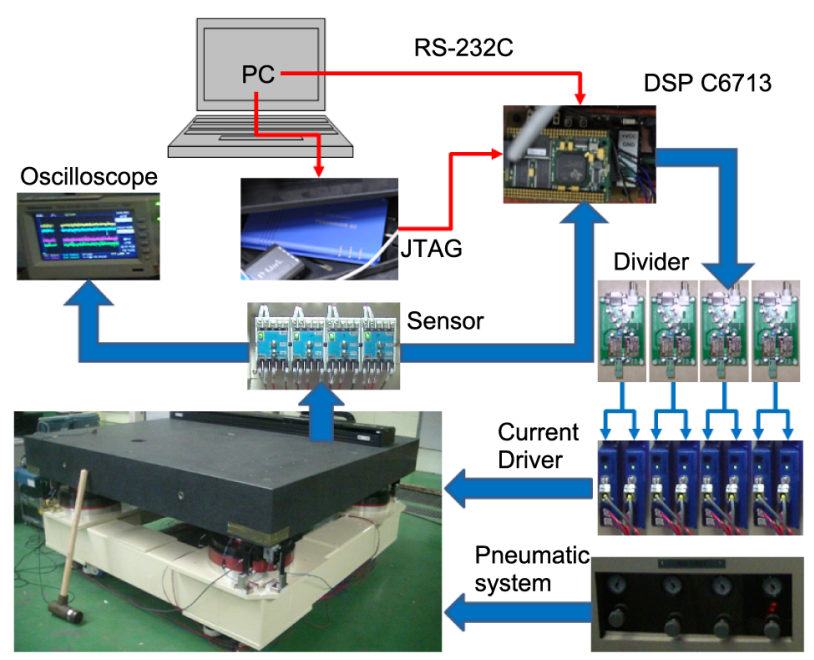

Figure 6. Control system in the air mount.

ners of the surface plate. The signal from the accelerometer was recorded and analyzed using a vibration measurement system (Muller-BBM PAK MKII). The reference level was obtained in the silent and static states in the first step.

The $\mathrm{P}$ gain was the sole parameter for tuning. Settling time was checked after impact on the surface place and then the $\mathrm{P}$ gain was increased. This procedure was repeated until unstable motion of the surface plate was observed. The $\mathrm{P}$ gain was chosen among the $\mathrm{P}$ gains experimented with the minimum settling time. The impulse, response was monitored under only pneumatic control in the second step. The electromagnetic control was activated and impulse response was recorded, in the last step.

\section{Results}

Signals measured by a gap sensor and an accelerometer are shown in Figures 7 and 8. In the graph, the horizontal axis is time, and the vertical is the displacement or the acceleration. The blue and red lines are the responses with and without control. A low pass filter of $50 \mathrm{~Hz}$ cut-off frequency was applied to the signals for noise in

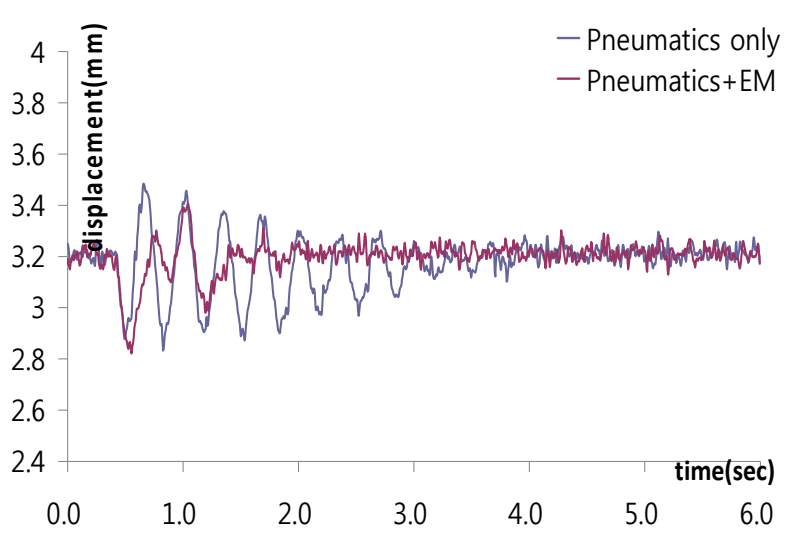

Figure 7. Impact responses measured by a gap sensor before and after EM control.

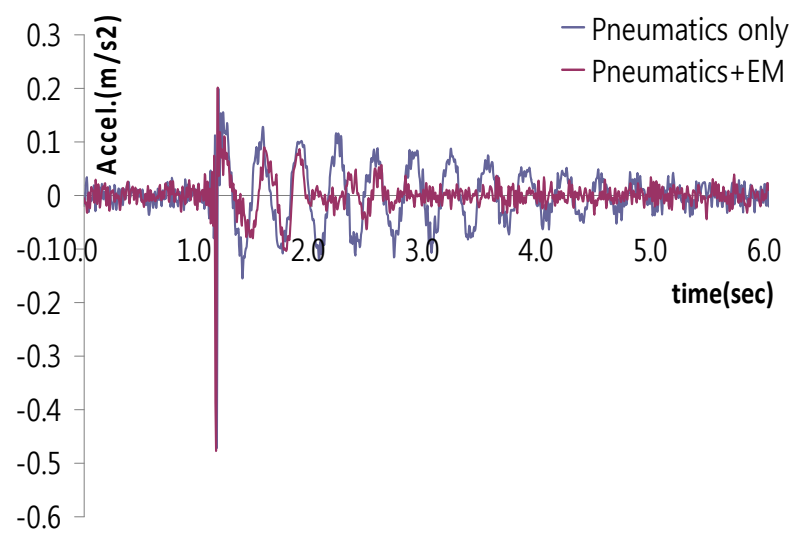

Figure 8. Impact responses measured by an accelerometer before and after EM control.

the test field. Before applying the electromagnetic control, oscillation caused by the impact continues for 3.4 sec, in the gap sensor and $4.1 \mathrm{sec}$ in the accelerometer. After activating the electromagnetic control, the settling time was reduced to $1.5 \mathrm{sec}$ in the gap sensor, and $1.7 \mathrm{sec}$ in the accelerometer. The settling time decreased approximately $55 \%$. The magnitudes of the first peak in both of the sensors did not decrease. This is the same trend as that in the report of our first model [7]. The first amplitudes of transient response with EM control were larger than those without EM. The first amplitude of displacement with control in the Figure 7 is slightly larger than that without control. This can be explained by the low pass filter and phase lag. The discrete low pass filter in the DSP had $40 \mathrm{~Hz}$ cut-off frequency, which can cause error between logical and actual position. The phase lag is caused by $\mathrm{AD}$ sampling, digital filtering, control logics and DA conversion. However, higher power is necessary to reduce the first amplitude, which is difficult to be generated by the EM. The large amplitude of acceleration in Figure 7 had negative sign, and is the kind of impulse caused by impact on the surface plate. The impulse is 
solid vibration, and its peak in the frequency domain is usually formed at the natural frequency of the surface plate. The amplitude is not motional vibration of the surface plate, and not the control target.

The time-domain signals were converted to frequency domain, using FFT. The frequency domain signals are shown in Figures 9 and 10. The Figure 9 shows the displacement response, and the Figure 10 does acceleration response. The horizontal axis is frequency and the vertical axis is the amplitude. In Figure 9, the first peak was formed at $1.2 \mathrm{~Hz}$ and the second peak at $3.0 \mathrm{~Hz}$ without control. The amplitude at the resonance frequency was reduced $-10 \mathrm{~dB}$ with control. In the Figure 10, one peak was formed at $3.0 \mathrm{~Hz}$, with and without control. The decrease with control was $-11 \mathrm{~dB}$, compared with the case without control.

The peaks in both of the sensors were at $3.0 \mathrm{~Hz}$ without the electromagnetic control. The theoretical natural frequency was $2.8 \mathrm{~Hz}$, so the measured data is reliable. After the electromagnetic control was applied, both of the peaks decreased below $-10 \mathrm{~dB}$.

In the time domain, the settling time was reduced, but the initial amplitude of the transient response was not

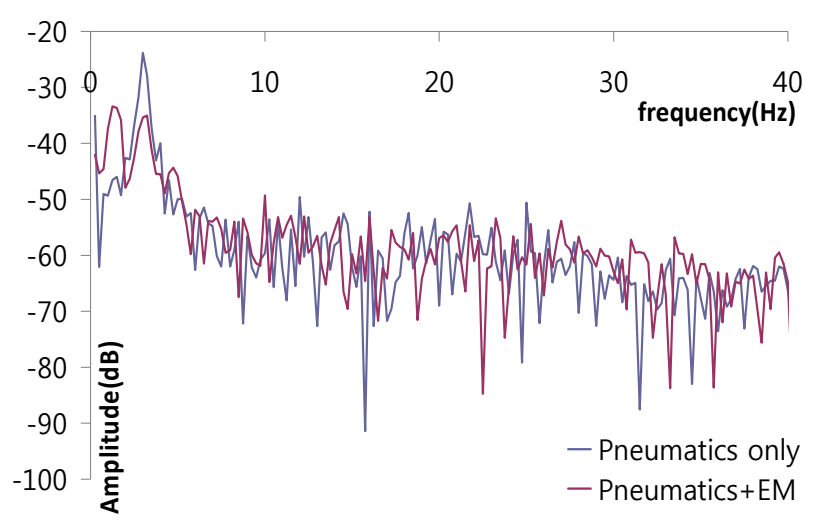

Figure 9. Frequency analysis measured by a gap sensor before and after EM control.

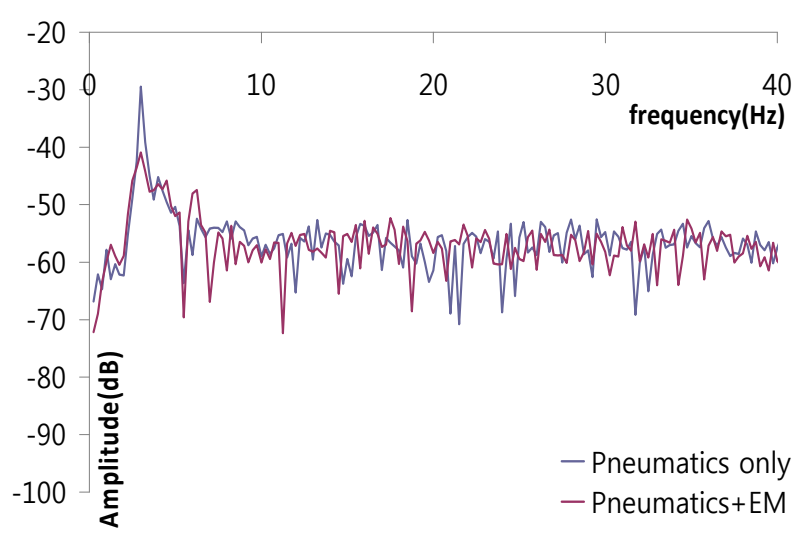

Figure 10. Frequency analysis measured by an accelerometer before and after EM control. because the electro-magnetic force is not so large as the pneumatic force. However, the resonance frequency was reduced in the frequency domain. This indicates that our device can prevent low frequency resonance and long oscillation.

\section{Conclusion}

We designed and constructed an active device, by combining pneumatic and electromagnetic forces for decreasing the low-frequency resonance in an isolator. The electromagnetic parts have dual stators and an armature, without any permanent magnets. The forces between the stators and the armature were simulated using FEM. The device was installed in an air mount used in manufacturing machines. A DSP-based control system was connected to the devices. Impulse responses were recorded and analyzed in an impact test. The results show that the settling time can be reduced to $55 \%$, and the amplitude of the resonance frequency decreases $-10 \mathrm{~dB}$.

\section{Acknowledgements}

This paper was presented to the international Congress on Sound and Vibration, 2012, Vilnius, Lithuania. The authors, appreciate the technical support provided by RMS Technology (http://www.rmstech.co.kr) in this research.

\section{REFERENCES}

[1] D. K Han and P. H. Chang, "A Robust Two-Time-Scale Control Design for a Pneumatic Vibration," Proceedings of 46th IEEE Conference on Decision and Control, New Orleans, 12-14 December 2007, pp. 1666-1672.

[2] Y. C. Zhong, Q. J. Yang and G. Bao "Nonlinearity Analyses of Pneumatic Vibration Isolation System with Simple Harmonic Excitations," Proceedings of 3rd IEEE Computer Science and Information Technology, Chengdu, 9-11 July 2010, pp. 814-817.

[3] P. H. Chang, D. K. Han, Y. H. Shin and K. J. Kim "Effective Suppression of Pneumatic Vibration Isolators by Using Input-Output Linearization and Time Delay Control," Journal of Sound and Vibration, Vol. 329, No. 10, 2010, pp. 1636-1652.

[4] L. Zuo and J. E. Slotine, "Effective Robust Vibration Isolation via Frequency-Shaped Sliding Control and Modal Decomposition," Journal of Sound and Vibration, Vol. 285, No. 4-5, 2005, pp. 1123-1149.

[5] T. Mizuno, M. Takasaki, D. Kishita and K. Hirakawa, "Vibration Isolation System Combining Zero Power Magnetic Suspension with Springs," Control Engineering Practice, Vol. 15, No. 2, 2007, pp. 187-196.

[6] D. Jones and R. Owen, "A Magnetically Levitated AntiVibration Mount," IEEE Transactions on Magnetics, Vol. 20, No. 5, 1984, pp. 1687-1689.

[7] H. T. Kim, C. H. Kim, K. W. Lee, G. S. Lee and S. W. 
Son, "An Electro-Magnetic Air Spring for Vibration Control in Semiconductor Manufacturing," Transactions of the KSNVE, Vol. 20, No. 12, 2010, pp. 1128-1138.

[8] H. T. Kim, C. H. Kim, S. B. Kang, K. W. Lee, J. H. Baek and H. H. Han, "Control of Air Mount Combined with Electro-Magnetics and MR," Proceedings of the KSNVE Annual Spring Conference, 2011, pp. 27-29.

[9] H. T. Kim, C. H. Kim, S. B. Kang, K. W. Lee, J. H. Baek and H. H. Han, "A 3 DOF Model for an Electro Magnetic Air Mount," Advances in Acoustics and Vibration, Vol.
2012, 2012, Article ID: 218429.

[10] K. Watanabe, Y. Kanemitsu, N. Haga and M. Suzuki, "Vibration Resistance Device," Japan Patent No. 07353956, 1995.

[11] S. J. Moon, S. H. Park, J. A. Jeong, Y. C. Huh, C. H. Kim and S. M. Choi, "A Study on the Development of a Hybrid Electromagnetic Actuator Against Microvibration," Transactions of the KSNVE, Vol. 21, No. 5, 2011, pp. 475-483. 